\title{
BURNUP SENSITIVITY CALCULATIONS WITH CBZ FOR LIGHT WATER REACTOR ASSEMBLY PROBLEMS
}

\author{
Go Chiba ${ }^{1}$ \\ ${ }^{1}$ Faculty of Engineering, Hokkaido University \\ Kita 13 Nishi 8, Sapporo, 060-8628, Japan \\ go_chiba@eng.hokudai.ac.jp
}

\begin{abstract}
Sensitivities of $k_{\infty}$ and nuclides number densities during nuclear fuel burnup with respect to nuclear data are calculated with a reactor physics code system CBZ. Sensitivity calculations are carried out with the depletion perturbation theory applicable to nuclear fuel assemblies including burnable absorbers. Numerical results are presented both for BWR and PWR assemblies, and those demonstrate usefulness and effectiveness of burnup sensitivity calculation capabilities for LWR fuel assemblies.
\end{abstract}

KEYWORDS: depletion perturbation theory, sensitivity, fuel assembly, predictor-corrector method

\section{INTRODUCTION}

Uncertainty quantification of reactor physics parameters/related quantities and data assimilation using measurable parameters/quantities have become important subjects to ensure reliability of numerical predictions and to reduce uncertainty of these predictions in the field of reactor physics. Although the stochastic (random sampling) approach[1] is becoming a standard method to perform this by virtue of significant advancement of computer powers, the classical perturbation theory-based approach[2] is sometimes convenient, and the role of this in the reactor physics field still remains. Our research group at Hokkaido University has developed our own reactor physics code system named CBZ, which is applicable for wide range of reactor physics problems, such as whole-core burnup calculations of fast neutron systems, assembly burnup calculations of thermal neutron systems, neutron transport calculations for fusion neutronics, etc. One of important capabilities of the CBZ code system is sensitivity calculations of nuclear fuel burnup-related parameters/quantities with respect to nuclear data[3]. This capability is based on the generalized perturbation theory for nuclear fuel burnup problems, the depletion perturbation theory, which has been established in the past by researchers such as Gandini[4] and Williams[5]. Our research group has implemented sensitivity calculation capability based on this theory into the CBZ code system, and light water reactor (LWR) fuel assemblies including burnable absorbers can be handled with at present. To mitigate time-discretization errors in fuel assembly burnup problems, some advanced numerical schemes such as the predictor-corrector (PC) method are mandatory, and the depletion perturbation theory applicable to the $\mathrm{PC}$ method has been also established during our previous work[6].

In the present paper, we will show numerical results about sensitivities of reactor physics parameters/related quantities to nuclear data in LWR fuel assembly problems. Sensitivities of infinite 
neutron multiplication factors $k_{\infty}$ at several different burnup and sensitivities of fuel pin-wise nuclide inventory can be calculated with the new capability of CBZ. With this capability, more accurate and reliable uncertainty quantification and data assimilation using measurement data about nuclide inventory become possible since sensitivities of nuclide inventory can be calculated not in a simplified model such as pin-cell model but in full-assembly model.

\section{DEPLETION PERTURBATION THEORY WITH PREDICTOR-CORRECTOR METHOD}

Let us consider a nuclear fuel burnup calculation, and nuclides number densities of this fuel at time $t$ are represented by a vector as $\mathbf{N}(t)$. We assume that an initial condition of the nuclides number density is provided at $t_{0}$. Usually we divide the whole depletion time period into several time steps, and a burnup matrix is assumed constant during each time step. When we divide the period into $I$ time steps, the following burnup equation can be defined:

$$
\frac{d \mathbf{N}(t)}{d t}=\mathbf{M}_{i} \mathbf{N}(t), \quad\left(t_{i-1} \leq t \leq t_{i}, \quad i=1,2, \ldots, I\right),
$$

where $\mathbf{M}_{i}$ is a burnup matrix of step $i$, and is defined from microscopic cross sections and neutron flux at $t_{i}$. The neutron flux at $t_{i}, \boldsymbol{\Phi}_{i}$, is defined from the following neutron transport equation:

$$
\mathbf{B}_{i} \boldsymbol{\Phi}_{i}=0,
$$

where the operator $\mathbf{B}_{i}$ is defined from $\mathbf{N}\left(t_{i-1}\right)$. When the predictor-corrector (PC) method is adopted, burnup calculation procedure in each step is modified as follows. At a predictor step, the following procedure, which is the exactly same as the conventional one, is adopted:

$$
\begin{gathered}
\mathbf{B}_{i}^{p} \boldsymbol{\Phi}_{i}^{p}=0, \\
\frac{d \mathbf{N}^{p}(t)}{d t}=\mathbf{M}_{i}^{p} \mathbf{N}^{p}(t) .
\end{gathered}
$$

Then with the transport operator $\mathbf{B}_{i}^{c}$ defined from $\mathbf{N}^{p}\left(t_{i}\right)$, a corrector step is carried out as follows:

$$
\begin{gathered}
\mathbf{B}_{i}^{c} \boldsymbol{\Phi}_{i}^{c}=0, \\
\frac{d \mathbf{N}^{c}(t)}{d t}=\mathbf{M}_{i}^{c} \mathbf{N}^{c}(t) .
\end{gathered}
$$

Note that a burnup matrix $\mathbf{M}_{i}^{x}$ is defined from $\boldsymbol{\Phi}_{i}^{x}(x=p, c)$. Finally nuclides number density at the end of time step, $t_{i}$, is calculated as an average of $\mathbf{N}^{p}\left(t_{i}\right)$ and $\mathbf{N}^{c}\left(t_{i}\right)$.

A sensitivity of number density of nuclide $j$ at $t_{I}, N_{j}\left(t_{I}\right)$, with respect to a nuclear data $\sigma$ can be calculated with the following equation based on the depletion perturbation theory with the PC method[6].

$$
\frac{d N_{j}\left(t_{I}\right)}{d \sigma}=\sum_{i=1}^{I}\left(I_{i}^{p}+I_{i}^{c}\right)
$$

where

$$
I_{i}^{x}=\int_{t_{i-1}}^{t_{i}} \mathbf{w}^{x T}(t) \frac{\partial \mathbf{M}_{i}^{x}}{\partial \sigma} \mathbf{N}^{x}(t) d t+\left\langle\boldsymbol{\Gamma}_{i}^{x \dagger} \frac{\partial \mathbf{B}_{i}^{x}}{\partial \sigma} \boldsymbol{\Phi}_{i}^{x}\right\rangle, \quad(x=p, c) .
$$


Note that it is assumed here that neutron flux normalization does not depend on any reaction cross sections for simplicity.

In Eq. (8), a vector $\mathbf{w}^{x}(t)$ is adjoint number densities, and is defined as

$$
\frac{d \mathbf{w}^{x}(t)}{d t}=-\mathbf{M}_{i}^{x T} \mathbf{w}^{x}(t), \quad\left(t_{i-1}<t<t_{i}\right),
$$

with a final condition $\mathbf{w}^{x}\left(t_{I}\right)=\mathbf{e}_{j}$. Note that $\mathbf{e}_{j}$ is a vector in which the $j$ th entry is unity and others are zero. At boundaries between subsequent time steps, discrete changes known as jump condition should be considered for $\mathbf{w}^{c}(t)$, but its detail is omitted here and can be found in Ref. [6]. A generalized adjoint neutron flux represented as $\Gamma_{i}^{x \dagger}$ in Eq. (8) is defined as

$$
\mathbf{B}_{i}^{x \dagger} \boldsymbol{\Gamma}_{i}^{x \dagger}=\mathbf{S}_{i}^{x \dagger}
$$

Detail of the source term $\mathbf{S}_{i}^{x \dagger}$ is also omitted here.

Sensitivity of $k_{\infty}$ at $t_{I}$ to $\sigma$ can be calculated as follows:

$$
\frac{d k_{\infty}}{d \sigma}=\frac{\partial k_{\infty}}{\partial \sigma}+\frac{\partial k_{\infty}}{\partial \boldsymbol{\Phi}_{I}} \cdot \frac{d \boldsymbol{\Phi}_{I}}{d \sigma}+\frac{\partial k_{\infty}}{\partial \mathbf{N}\left(t_{I}\right)} \cdot \frac{d \mathbf{N}\left(t_{I}\right)}{d \sigma} .
$$

The first and second terms in right-hand-side of this equation can be easily calculated with the classical perturbation theory. The third term is rewritten as

$$
\frac{\partial k_{\infty}}{\partial \mathbf{N}\left(t_{I}\right)} \cdot \frac{d \mathbf{N}\left(t_{I}\right)}{d \sigma}=\sum_{j}\left\{\frac{\partial k_{\infty}}{\partial N_{j}\left(t_{I}\right)} \cdot \frac{d N_{j}\left(t_{I}\right)}{d \sigma}\right\} \approx \frac{d}{d \sigma}\left\{\sum_{j} \frac{\partial k_{\infty}}{\partial N_{j}\left(t_{I}\right)} N_{j}\left(t_{I}\right)\right\} .
$$

This quantity can be calculated with the depletion perturbation theory with a final condition of $w_{j}^{x}\left(t_{I}\right)=\frac{\partial k_{\infty}}{\partial N_{j}\left(t_{I}\right)}$.

Note that all the sensitivities shown in the following sections are relative ones: $\left(\frac{d N_{j}}{d \sigma}\right) \cdot\left(\frac{\sigma}{N_{j}}\right)$ and $\left(\frac{d k_{\infty}}{d \sigma}\right) \cdot\left(\frac{\sigma}{k_{\infty}}\right)$.

\section{PROBLEMS SPECIFICATION}

In the present study, sensitivities of $k_{\infty}$ and number density of americium-241 with respect to some reaction cross sections in BWR and PWR fuel assemblies are calculated with the depletion perturbation theory as mentioned above.

As a BWR assembly, we use an assembly model proposed in the OECD/NEA burnup credit benchmark phase III-C[7]. The geometrical specification is shown in Fig. 1. Three cases with different void ratios, $0 \%, 40 \%$ and $70 \%$, are considered.

As a PWR assembly, we use the assembly model constructed in our previous work[8]. Its geometrical specification is shown also in Fig. 1. Note that $\mathrm{Gd}_{2} \mathrm{O}_{3}$ rods are composed of $9.6 \mathrm{wt} \%$ 
$\mathrm{Gd}_{2} \mathrm{O}_{3}$ and $\mathrm{UO}_{2}$ whose uranium-235 enrichment is $2.6 \mathrm{wt} \%$. Gadolinium concentration of the PWR assembly is higher than that of the BWR assembly (5.0 wt $\%$ ).

In burnup calculations, neutron flux is normalized by total power; neutron flux normalization effect on sensitivities is taken into account in results shown in the following sections although its theory is not mentioned in the preceding section.
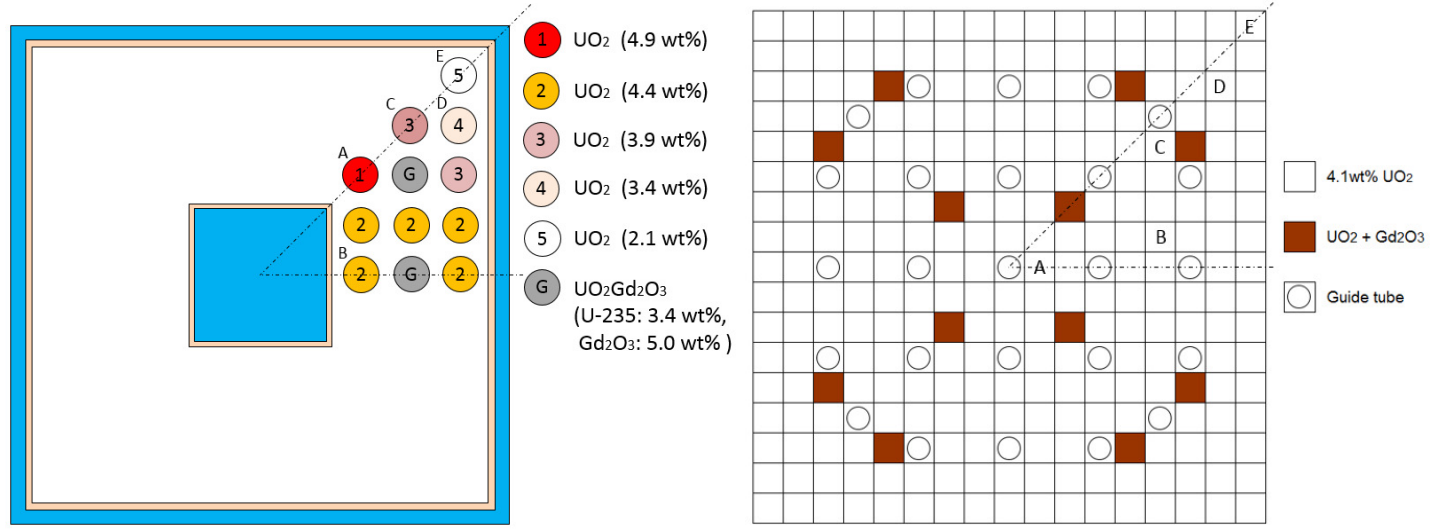

Figure 1: Geometric configurations of BWR and PWR fuel assemblies (left: BWR, right: PWR)

\section{NUMERICAL PROCEDURE}

All the calculations are carried out with a module named MulticellBurner in a deterministic reactor physics code system CBZ, which is under development at Hokkaido University. 107-group self-shielded cross sections are calculated based on the advanced Bondarenko model[9] from a 107-group CBZLIB generated from JENDL-4.0. Lattice effect is taken into account by the Dancoff factor method applicable for arbitrary geometry like fuel assemblies[10]. Neutron transport equation is solved by a module MEC based on the method of characteristics. Scattering anisotropy is taken into account by the P0 transport approximation. With neutron flux and self-shielded cross sections, fuel burnup calculations are carried out. A simplified burnup chain consisting of 138 fission products is used[11]. The burnup equation is solved by the matrix exponential method, and the matrix exponential is calculated by the mini-max polynomial approximation method[12,13]. Note that the implicit effect on the effective cross sections is neglected in sensitivity calculations.

\section{NUMERICAL RESULTS}

\subsection{Sensitivities of $k_{\infty}$}

First $k_{\infty}$ calculated by CBZ/MulticellBurner is shown in Fig. 2. Comparisons with well-verified continuous-energy Monte Carlo solutions have been made, and good agreement with these references has been confirmed.

Sensitivities of $k_{\infty}$ to $(\mathrm{n}, \gamma)$ cross section of uranium-238 in the BWR assembly at several different burnup/void ratios are shown in Fig. 3. Absolute values of sensitivities at $6.4 \mathrm{GWD} / \mathrm{t}$ are generally smaller than those at $12.8 \mathrm{GWD} / \mathrm{t}$ since neutron absorption of gadolinium isotopes would be 
(a) BWR

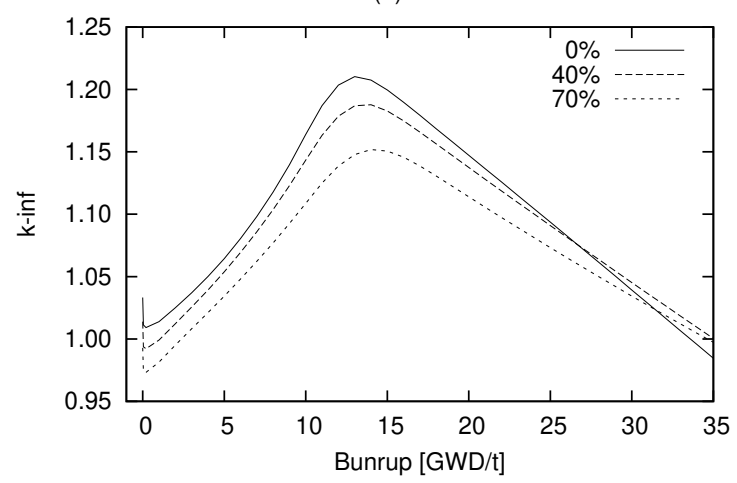

(b) PWR

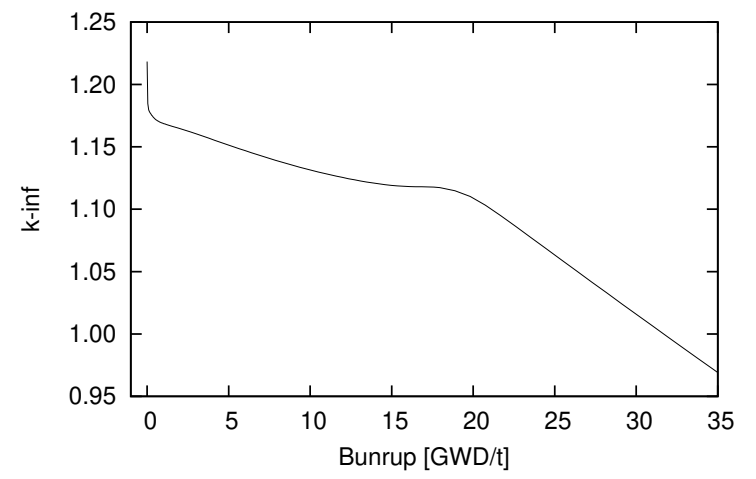

Figure 2: $k_{\infty}$ calculated with CBZ/MulticellBurner

significant at $6.4 \mathrm{GWD} / \mathrm{t}$. As fuel burnup increases from $12.8 \mathrm{GWD} / \mathrm{t}$, sensitivities are shifted to positive side, and some become positive in several energy groups. This is due to the burnup effect on sensitivities; if $(\mathrm{n}, \gamma)$ cross section of uranium-238 increases, generation of plutonium-239 is enhanced and positive effect to $k_{\infty}$ is expected. It is also shown that sensitivities in the epithermal range become absolutely large when void ratio increases because of neutron flux energy spectrum hardening.

Next comparisons are made in $k_{\infty}$ sensitivities between the PWR and BWR assemblies at the same burnup. Figure 4 shows sensitivities of $k_{\infty}$ to $(\mathrm{n}, \gamma)$ cross section of uranium-238 at $19.2 \mathrm{GWD} / \mathrm{t}$ and 32.0 GWD/t. Sensitivity profile in the PWR assembly shows similar shape with those in the BWR assembly.

\subsection{Sensitivities of number density of americium-241}

In this subsection, sensitivities of number density of americium-241 are calculated at different pin locations. The number density of americium-241 is chosen because this nuclide is generated through several different physical phenomena including neutron capture reactions and radioactive decay.

Figures 5 and 6 show sensitivities of americium-241 number density at $19.2 \mathrm{GWD} / \mathrm{t}$ to $(\mathrm{n}, \gamma)$ cross sections of americium-241 and uranium-238 with different void ratios in the BWR assembly. Pin locations are denoted to as A, B, C, D and E, and those are presented in Fig. 1. The uranium-235 enrichment is the highest in the pin A and the lowest in the pin E. High uranium-235 enrichment causes neutron flux energy spectrum hardening, and this effect can be seen in sensitivities in thermal energy range. Also it is shown that dependence of sensitivities on pin location and composition becomes significant in the epithermal range when void ratio is large.

Figure 7 shows sensitivities of americium-241 number density at different pin locations in the PWR assembly. As expected, dependence of sensitivities on pin location are small in comparison with those in the BWR assembly. 
(a) $0 \%$

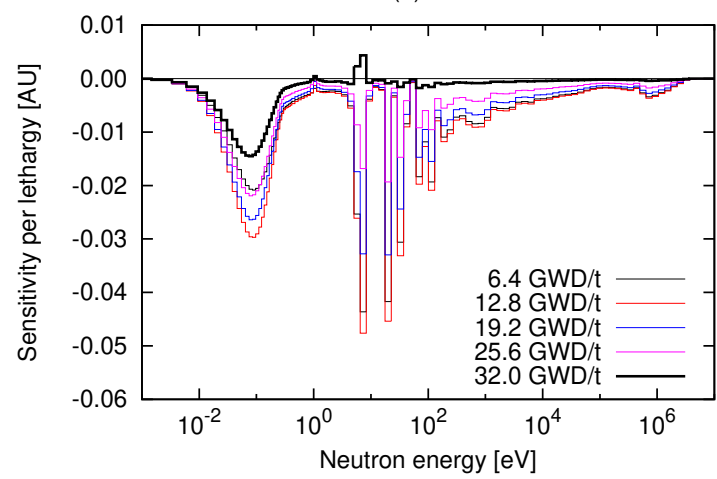

(b) $40 \%$

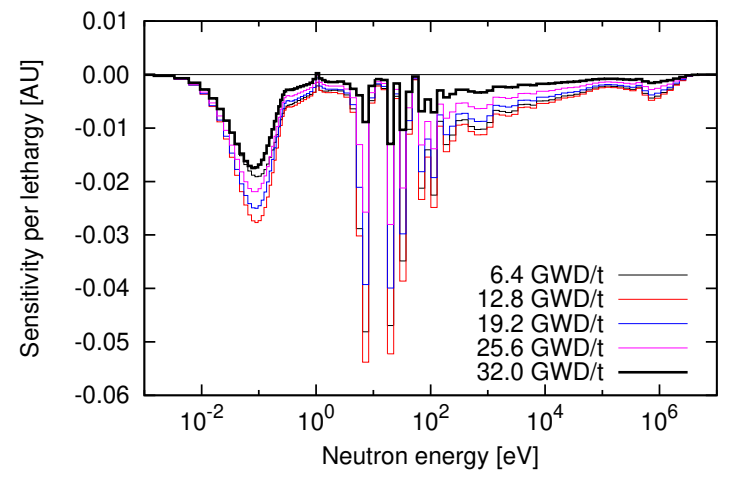

(c) $70 \%$

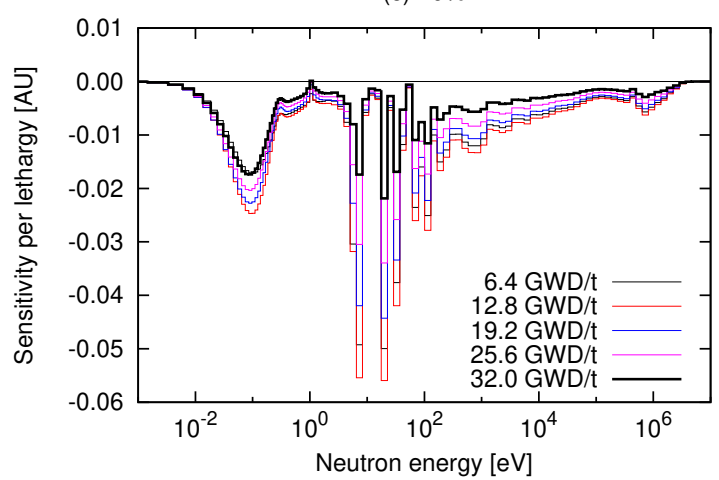

Figure 3: Sensitivity of $k_{\infty}$ to $(\mathbf{n}, \gamma)$ cross section of uranium-238 in the BWR assembly

(a) $19.2 \mathrm{GWD} / \mathrm{t}$

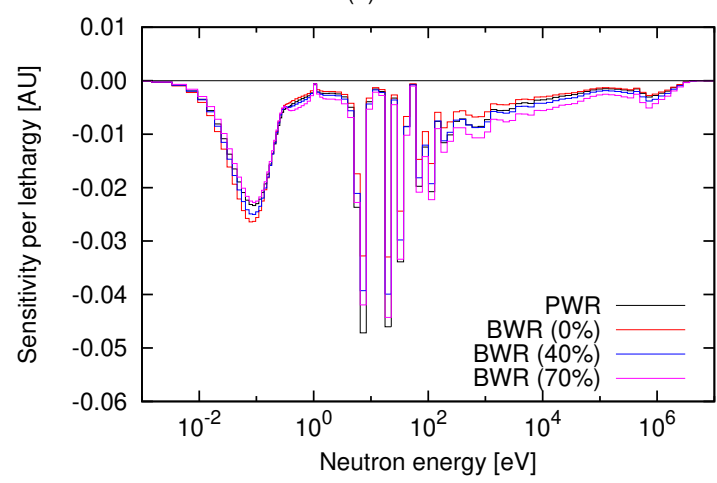

(b) $32.0 \mathrm{GWD} / \mathrm{t}$

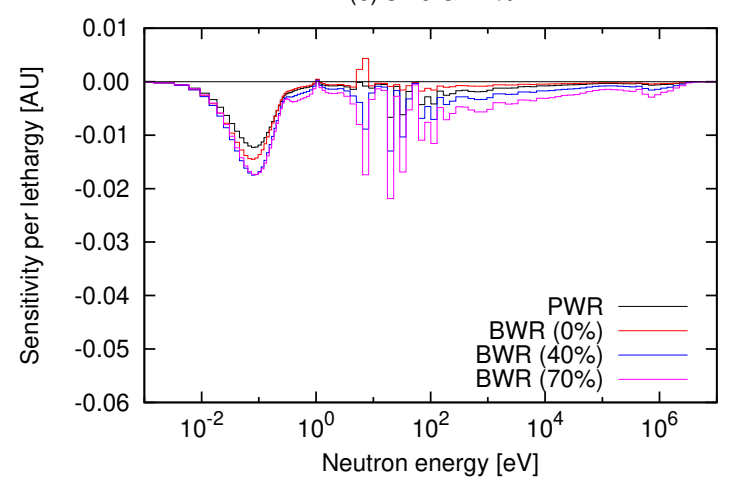

Figure 4: Sensitivity of $k_{\infty}$ to $(\mathrm{n}, \gamma)$ cross section of uranium-238 in the BWR and PWR assemblies

\section{CONCLUSIONS}

Sensitivities of $k_{\infty}$ and nuclides number densities during nuclear fuel burnup with respect to nuclear data have been calculated with a reactor physics code system CBZ, and some numerical 
(a) Am-241 (n,g)

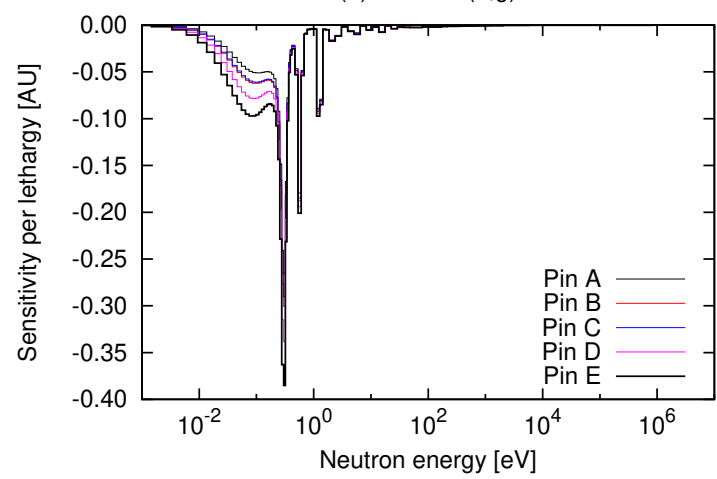

(b) U-238 (n,g)

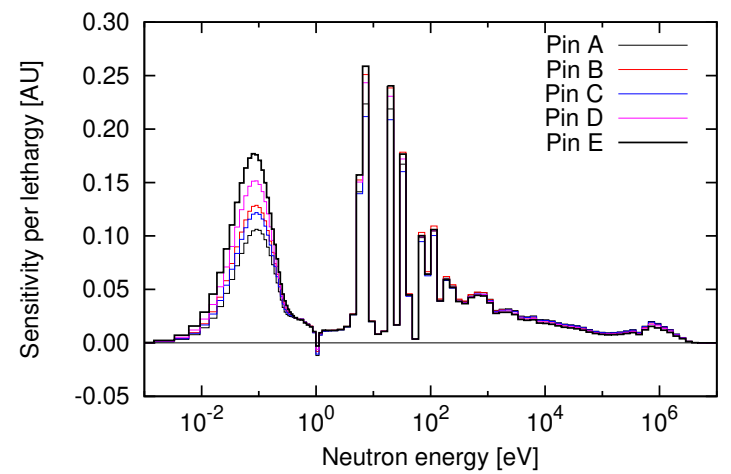

Figure 5: Sensitivity of Am-241 number density at 19.2 GWD/t to $(\mathrm{n}, \gamma)$ cross section in the BWR assembly with void ratio of $0 \%$

(a) Am-241 (n,g)

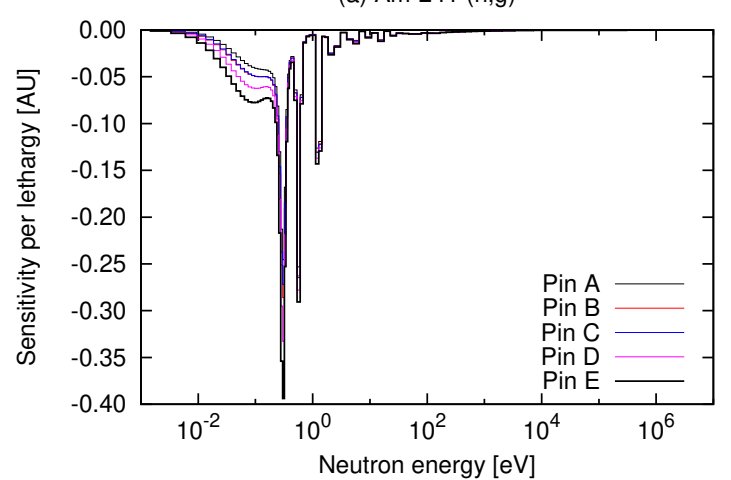

(b) U-238 (n,g)

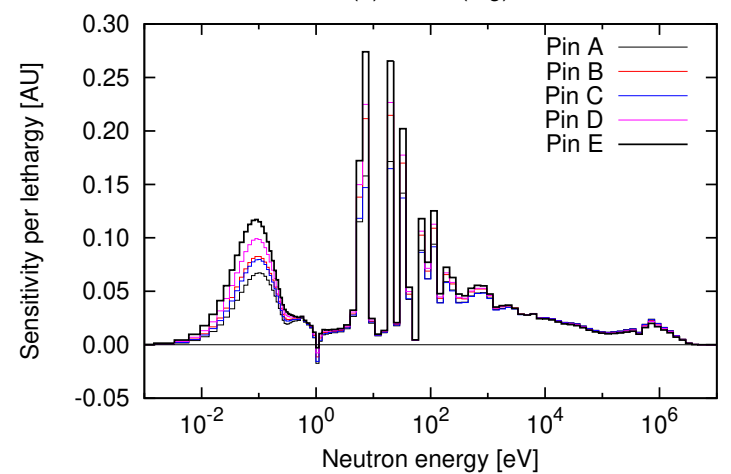

Figure 6: Sensitivity of Am-241 number density at 19.2 GWD/t to (n, $\gamma)$ cross section in the BWR assembly with void ratio of $70 \%$

results have been presented. Sensitivity calculations have been carried out with the depletion perturbation theory applicable to nuclear fuel assemblies including burnable absorbers. Numerical results have been presented both for the BWR and PWR assemblies, and some comparisons have been made focusing on differences in burnup, fuel assembly type between BWR and PWR, and void ratio in the BWR assemblies. The present calculations have demonstrated usefulness and effectiveness of burnup sensitivity calculation capabilities for LWR fuel assemblies.

\section{REFERENCES}

[1] M. D. McKay, "Sensitivity and Uncertainty Analysis Using a Statistical Sample of Input Values," Uncertainty Analysis, pp. 145-186, CRC Press (1988).

[2] D. G. Cacuchi, "The Forward and Adjoint Methods of Sensitivity Analysis," Uncertainty Analysis, pp. 71-144, CRC Press (1988). 
(a) Am-241 (n,g)

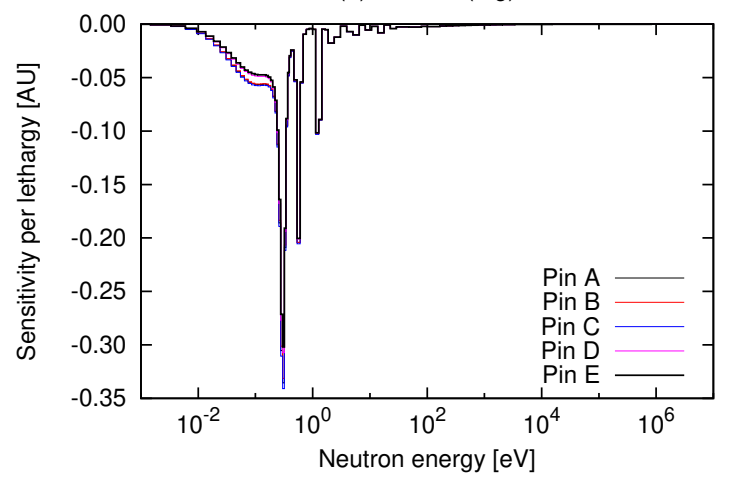

(b) $U-238(n, g)$

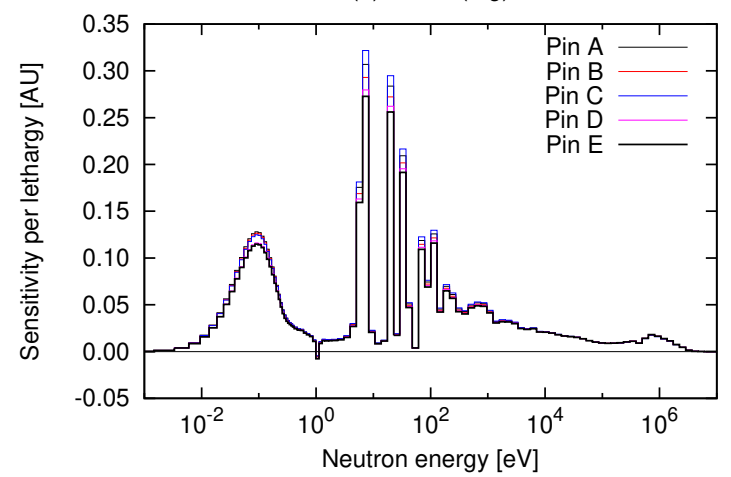

Figure 7: Sensitivity of Am-241 number density at 19.2 GWD/t to (n, $\gamma)$ cross section in the PWR assembly

[3] G. Chiba, Y. Kawamoto, T. Narabayashi, "Development of Fuel Depletion Sensitivity Calculation Module for Multi-Cell Problem in Deterministic Reactor Physics Code System CBZ," Ann. Nucl. Energy, 96, pp. 313-323 (2016).

[4] A. Gandini, "A Method of Correlation of Burnup Measurements for Physics Prediction of Fast Power-Reactor Life," Nucl. Sci. Eng., 38, pp. 1-7 (1969).

[5] M. L. Williams, "Development of Depletion Perturbation Theory for Coupled Neutron/Nuclide Fields," Nucl. Sci. Eng., 70, pp. 20-36 (1979).

[6] G. Chiba, "Perturbation Theory for Nuclear Fuel Depletion Calculations with PredictorCorrector Method," J. Nucl. Sci. Technol., 55, pp. 290-300 (2018).

[7] "Burn-up Credit Criticality Safety Benchmark Phase III-C," OECD/NEA, NEA/NSC/R/(2015)6.

[8] S. Okumura, G. Chiba, "Development of Nuclear Fuel Depletion Calculation Capability for LWR Fuel Assembly in Reactor Physics Code System CBZ," Proc. of Reactor Physics Asia, RPHA2017, Chengdu, China, August 24-25, 2017 (2017).

[9] G. Chiba, T. Narabayashi, "Advanced Bondarenko Method for Resonance Self-Shielding Calculations in Deterministic Reactor Physics Code System CBZ," Ann. Nucl. Energy, 96, pp. 277-286 (2016).

[10] N. Sugimura, A. Yamamoto, "Evaluation of Dancoff Factors in Complicated Geometry Using the Method of Characteristics," J. Nucl. Sci. Technol., 43, pp. 1182-1187 (2006).

[11] G. Chiba, M. Tsuji, T. Narabayashi, Y. Ohoka, T. Ushio, "Important Fission Products Identification Method for Simplified Burnup Chain Construction," J. Nucl. Sci. Technol., 52, pp. 953-960 (2015).

[12] Y. Kawamoto, G. Chiba, M. Tsuji, T. Narabayashi, "Numerical Solution of Matrix Exponential in Burn-up Equation Using Mini-Max Polynomial Approximation," Ann. Nucl. Energy, 80, pp. 219-224 (2015).

[13] G. Chiba, Y. Ohoka, K. Yamamoto, H. Nagano, "Revisiting Mini-Max Polynomial Approximation Method for Nuclear Fuel Depletion Calculations," Proc. of Int. Conf. on Math. Comp. Methods Applied to Nucl. Sci. Eng., M\&C2019, Portland, OR, August 25-29, 2019 (2019). 